\title{
ADVERSE EFFECTS INDUCED BY ACTIVITIES DEVELOPED IN THE MUNICIPAL WASTEWATER TREATMENT PLANTS IN THE PROXIMITY AREAS
}

\author{
Adriana Cuciureanu $^{1}$, Bogdan Stanescu $^{1}$, Lidia Kim ${ }^{1}$
}

${ }_{1}$ National Research and Development Institute for Industrial Ecology, Drumul Podu Dambovitei Street, no. 71- 73, Bucharest, postal code: 060652, ecoind@incdecoind.ro, Romania,

\begin{abstract}
Among the many environmental problems facing the society one of the most important is undoubtedly a municipal wastewater treatment. Municipal wastewater treatment plant removes the pollutants from urban wastewater composed of a mixture of domestic wastewater or domestic wastewater mixed with industrial wastewater and / or pluvial waters runoff. Wastewater treatment is beneficial by solving a series of problems that affect the environment but in some cases, contributes to the generating an environmental impact near the sites, considering that policy instruments are improperly applied. The paper presents the results of investigations carried on the quality of soil and water in the areas surrounding of the two municipal wastewater treatment plants, chosen as case studies, from different regions of the country. For those have been achieved diagrams representing the state of pollution in the selected areas. The impact assessment induced in the vicinity of the activities carried out in sewage treatment plants was achieved by interpreting the results of investigations in compliance with specific legislation
\end{abstract}

Keywords: areas, assessment, pollution, proximity, wastewater treatment plants

\section{Introduction}

Problem of quality and environmental protection came into actuality as the world has become aware of the need for conservation and more efficient use of the productive potential of the environment. Therefore both global and national attention is given increasingly higher environmental activities and supervision of changes to the quality [1].

The activities of the municipal wastewater treatment plants are authorized in terms of environmental protection in accordance with environmental legislation specifies [2].

The activities developped by adverse conditions in a municipal wastewater treatment plants can sometimes lead to deterioration of the quality environmental components of the site and can be extended in their proximity.

Negative effects induced on the quality of environmental factors that can extend and proximity sites are generated by: the occurrence of sections of damaged sewerage system wastewater collectors, the discharge of insufficiently treated in natural emissary, diffuse emissions from the handling of powdery substances and products or volatility, etc.

Nationwide, as a result of the enforcement of environmental permits, quality monitoring is done in site environmental components [3], but it not enough however highlighted the effects that may occur in the vicinity of the sites. 


\section{Materials and Methods (or Experimental)}

It was followed analysis of two wastewater treatment plants with different particularities in different development regions of Romania selected as case studies: South-East Region 2 - WWTP of Focsani and South Muntenia Region 3 - WWTP Slobozia.

Measuring the impact of induced by the activities carried out in the two treatment plants in proximity locations was conducted in two campaigns seasonal: summer and autumn. For both municipal wastewater treatment plants (Focsani and Slobozia) methodology was applied to investigate the environmental component soil, wastewater and surface water quality, as follows [4]:

a) The investigation of field was achieved by:

- direct observations during visits to sites

- identifying how to use the land in the vicinity of the stations

- defining the experimental field, respectively establish the locations of sampling soil and water

- sampling and appropriate conservation

- registration of the geographical position of sampling points

b) Laboratory investigation was carried out by:

- characterization of soil and water samples taken in terms of global and specific indicators

- applying standardized test methods for determining quality indicators select and use of the latest generation analytical equipment

In the investigation field activities for each of the treatment plant were established sampling locations outside the site boundary. They were distributed in order to cover a larger area, thus defining the experimental field. We used a manual drilling equipment, Eijkelkamp probe, samples are collected in glass jars, with lids tightly closed. The soil samples were taken from each neighborhood treatment plants, from $0-10 \mathrm{~cm}$ and $30-40 \mathrm{~cm}$ in profile each having 8 / location.

Investigation of water quality has been achieved by taking in each location, discharge water into the station and surface water samples (emissary receptor effluent treatment station - Putna river where wastewater treatment plant in Focsani and lalomita river where wastewater treatment plant Slobozia), upstream and downstream of the point of discharge of the effluent station. Sampling of water (wastewater and surface) were used telescopic equipment type scooper.

The geographic location of all sampling points was achieved with a Garmin GPS receiver.

During the investigation in laboratory activities for each treatment plant, we were determined the quality indicators:

- for soil samples: $\mathrm{pH}$, wet, organic carbon, total nitrogen, ammonium, chloride, total phosphorus, sulphates, nickel, zinc, iron, copper, cadmium

-for water samples: $\mathrm{pH}, \mathrm{COD}, \mathrm{BOD}$, ammonia, total nitrogen, total phosphorus, nitrates, chlorides, sulfides, filterable residue, nickel, copper, zinc, iron, cadmium.

For WWTP Focsani: July samples are denoted S1 $\div$ S4 and the September $S^{\prime} 1 \div S^{\prime} 4$.

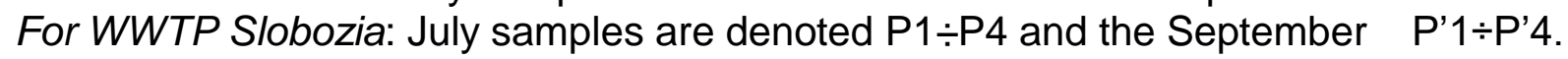

Location of sampling points is shown in the following figures. 

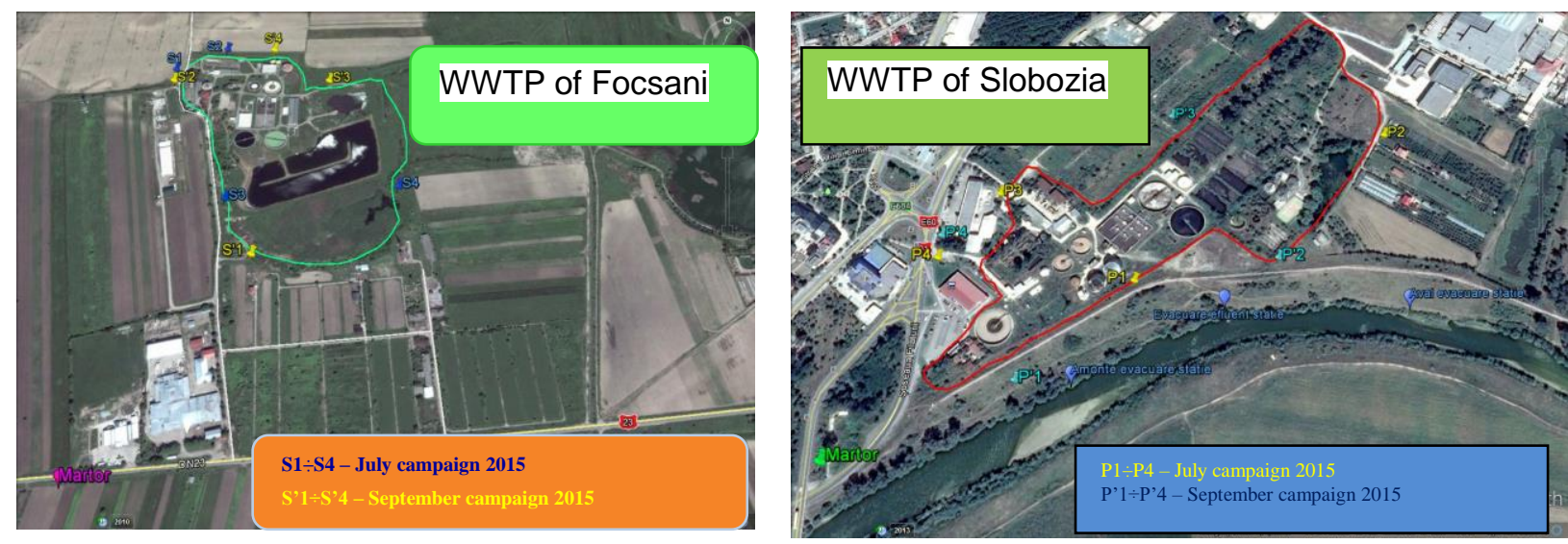

\section{Results and Discussion}

The results of characterization of soil samples taken from both neighborhoods treatment plants are presented in Tables 1,2, 3, 4 and the characterization of water samples are presented in Tables 5.6.

Table 1 Characterization of soil samples near the wastewater treatment plant of Focsani - July 2015 campaign

\begin{tabular}{|c|c|c|c|c|c|c|c|c|c|c|}
\hline \multirow[t]{2}{*}{ Quality Indicator } & \multicolumn{10}{|c|}{ Sample } \\
\hline & $\mathbf{S 1 / 1}$ & S1/2 & $\mathrm{S} 2 / \mathbf{1}$ & $\mathrm{S} 2 / 2$ & $\mathrm{~S} 3 / 1$ & $\mathrm{~S} 3 / 2$ & $S 4 / 1$ & $\mathrm{~S} 4 / 2$ & M/1 & $M / 2$ \\
\hline $\mathrm{pH}$ & 7,22 & 6,94 & 6,93 & 7,05 & 6,87 & 6,89 & 7,2 & 7,05 & 6,53 & 6,95 \\
\hline wet, \% & 14,8 & 15,11 & 6,48 & 7,17 & 10,99 & 12,43 & 8,51 & 9,06 & 13,35 & 15,12 \\
\hline C organic, $\%$ & 0,28 & 0,57 & 0,3 & 0,21 & 0,11 & 0,3 & 0,15 & 0,22 & 0,06 & 0,07 \\
\hline $\begin{array}{l}\text { Total nitrogen, \% } \\
\text { d.s. }\end{array}$ & 0,201 & 0,191 & 0,202 & 0,176 & 0,232 & 0,213 & 0,195 & 0,198 & 0,159 & 0,151 \\
\hline $\begin{array}{l}\text { Ammonium, } \\
\mathrm{mg} / \mathrm{kg} \text { d.s. }\end{array}$ & 19,92 & 18,05 & 21,21 & 18,85 & 16,79 & 16,14 & 18,20 & 17,96 & 20,97 & 20,02 \\
\hline $\begin{array}{l}\text { Chloride, } \quad \mathrm{mg} / \mathrm{kg} \\
\text { d.s. }\end{array}$ & 33,33 & 31,81 & 30,36 & 22,94 & 39,88 & 36,48 & 32,56 & 35,64 & 32,77 & 30,18 \\
\hline Sulfati, mg/kg d.s. & 109,15 & 177,87 & 152,9 & 157,27 & 221,32 & 134,74 & 127,5 & 135,12 & 216,96 & 146,08 \\
\hline $\begin{array}{l}\text { Total phosphorus, } \\
\% \text { d.s. }\end{array}$ & 0,078 & 0,065 & 0,046 & 0,037 & 0,068 & 0,056 & 0,045 & 0,055 & 0,05 & 0,04 \\
\hline Nickel, mg/kg d.s. & 12,3 & 10,8 & 15,4 & 12,6 & 37,8 & 40,3 & 21,5 & 18,8 & 10,6 & 10,8 \\
\hline Zinc, $\mathrm{mg} / \mathrm{kg}$ d.s. & 78,9 & 85,4 & 84,5 & 69,6 & 134 & 93,2 & 68,5 & 75,2 & 52,1 & 55,1 \\
\hline Iron, $\mathrm{mg} / \mathrm{kg}$ d.s. & 2980 & 2765 & 3150 & 3420 & 3766 & 3700 & 2500 & 2204 & 2164 & 2100 \\
\hline $\begin{array}{l}\text { Cooper, } \quad \mathrm{mg} / \mathrm{kg} \\
\text { d.s. }\end{array}$ & 18,5 & 17,9 & 18,5 & 16,3 & 43,4 & 34,8 & 20,1 & 19,6 & 16,1 & 15,8 \\
\hline $\begin{array}{l}\text { Cadmium, mg/kg } \\
\text { d.s. }\end{array}$ & 0,15 & 0,12 & 0,14 & 0,15 & 0,25 & 0,28 & 0,2 & 0,18 & 0,12 & 0,14 \\
\hline
\end{tabular}

Table 2 Characterization of soil samples near the wastewater treatment plant of Focsani - September 2015 campaign

\begin{tabular}{|c|c|c|c|c|c|c|c|c|}
\hline \multirow[t]{2}{*}{ Quality Indicator } & \multicolumn{8}{|c|}{ Sample } \\
\hline & $S^{\prime} 1 / 1$ & $S^{\prime} 1 / 2$ & $S^{\prime} 2 / 1$ & $S^{\prime} 2 / 2$ & $S^{\prime} 3 / 1$ & $S^{\prime} 3 / 2$ & $S^{\prime} 4 / 1$ & $S^{\prime} 4 / 2$ \\
\hline $\mathrm{pH}$ & 6,89 & 6,76 & 6,58 & 6,93 & 7,37 & 7,11 & 7,34 & 6,87 \\
\hline wet, \% & 15,02 & 9,65 & 8,15 & 16,81 & 1,16 & 26,47 & 14,9 & 9,8 \\
\hline C organic, $\%$ & 0,85 & 0,69 & 0,19 & 0,32 & 0,24 & 0,22 & 0,15 & 0,27 \\
\hline $\begin{array}{l}\text { Total nitrogen, \% } \\
\text { d.s. }\end{array}$ & 0,382 & 0.257 & 0,163 & 0,149 & 0,071 & 0,059 & 0,072 & 0,057 \\
\hline $\begin{array}{l}\text { Ammonium, } \mathrm{mg} / \mathrm{kg} \\
\text { d.s. }\end{array}$ & 48,95 & 35,53 & 38,97 & 38,54 & 61,35 & 53,68 & 59,36 & 47,07 \\
\hline $\begin{array}{l}\text { Chloride, } \quad \mathrm{mg} / \mathrm{kg} \\
\text { d.s. }\end{array}$ & 45,9 & 35,3 & 27,05 & 38,27 & 43 & 43,4 & 16,6 & 19,7 \\
\hline Sulfati, $\mathrm{mg} / \mathrm{kg}$ d.s. & 527,1 & 307,7 & 223,1 & 251,2 & 225,6 & 202,6 & 374,8 & 231,6 \\
\hline $\begin{array}{l}\text { Total phosphorus, } \\
\% \text { d.s. }\end{array}$ & 0,23 & 0,11 & 0,11 & 0,108 & 0,052 & 0,058 & 0,057 & 0,054 \\
\hline Nickel, $\mathrm{mg} / \mathrm{kg}$ d.s. & 24,18 & 17,14 & 33,8 & 34,1 & 28,4 & 27,9 & 21,9 & 25,7 \\
\hline Zinc, $\mathrm{mg} / \mathrm{kg}$ d.s. & 106 & 81,3 & 49,14 & 47,6 & 111,9 & 98,5 & 30,88 & 36,9 \\
\hline Iron, $\mathrm{mg} / \mathrm{kg}$ d.s. & 5612 & 5531 & 4870 & 4812 & 3620 & 3597 & 3918 & 3968 \\
\hline Cooper, $\mathrm{mg} / \mathrm{kg}$ d.s. & 66 & 36,8 & 24,04 & 21,3 & 34,5 & 30,18 & 15,9 & 18,2 \\
\hline $\begin{array}{l}\text { Cadmium, } \mathrm{mg} / \mathrm{kg} \\
\text { d.s. }\end{array}$ & 0,5 & 0,06 & $<0,02$ & $<0,02$ & 0,15 & 0,09 & $<0,02$ & $<0,02$ \\
\hline
\end{tabular}

Table 3 Characterization of soil samples near the wastewater treatment plant of Slobozia - July 2015 campaign

\begin{tabular}{|c|c|c|c|c|c|c|c|c|c|c|}
\hline \multirow{2}{*}{ Quality Indicator } & \multicolumn{10}{|c|}{ Sample } \\
\hline & P1/1 & P1/2 & $\mathbf{P} 2 / \mathbf{1}$ & $\mathbf{P} 2 / \mathbf{2}$ & P3/1 & $\mathbf{P} 3 / 2$ & $\mathbf{P 4 / 1}$ & $\mathbf{P} 4 / 2$ & $\mathrm{M} / \mathbf{1}$ & M/2 \\
\hline $\mathrm{pH}$ & 6,99 & 6,84 & 6,73 & 6,70 & 6,93 & 7,14 & 6,57 & 6,83 & 6,67 & 6,82 \\
\hline wet, $\%$ & 12,25 & 10,26 & 13,93 & 12,33 & 12,47 & 13,01 & 33,06 & 33,08 & 12,8 & 14,1 \\
\hline C organic, \% & 0,08 & 0,06 & 0,46 & 0,39 & 0,07 & 0,06 & 0,78 & 1,1 & 0,05 & 0,04 \\
\hline $\begin{array}{l}\text { Total nitrogen, \% } \\
\text { d.s. }\end{array}$ & 0,283 & 0,259 & 0,405 & 0,340 & 0,215 & 0,194 & 0,871 & 0,782 & 0,186 & 0,220 \\
\hline $\begin{array}{l}\text { Ammonium, } \\
\mathrm{mg} / \mathrm{kg} \text { d.s. }\end{array}$ & 21,02 & 19,04 & 20,14 & 18,69 & 21,17 & 19,18 & 26,71 & 25,26 & 15,42 & 16,16 \\
\hline $\begin{array}{l}\text { Chloride, } \quad \mathrm{mg} / \mathrm{kg} \\
\text { d.s. }\end{array}$ & 24,27 & 39,55 & 37,12 & 48,59 & 56,78 & 32,64 & 116,67 & 106,09 & 20,1 & 18,8 \\
\hline Sulfati, $\mathrm{mg} / \mathrm{kg}$ d.s. & 168,66 & 125,91 & 187,05 & 248,65 & 672,91 & 578,22 & 389,9 & 348,17 & 121,2 & 114,7 \\
\hline
\end{tabular}


INCD ECOIND - INTERNATIONAL SYMPOSIUM - SIMI 2016 “THE ENVIRONMENT AND THE INDUSTRY", PROCEEDINGS BOOK

\begin{tabular}{|l|c|c|c|c|c|c|c|c|c|c|}
\hline $\begin{array}{l}\text { Total phosphorus, } \\
\% \text { d.s. }\end{array}$ & 0,065 & 0,058 & 0,184 & 0,162 & 0,151 & 0,146 & 0,605 & 0,593 & 0,058 & 0,064 \\
\hline Nickel, mg/kg d.s. & 30,1 & 26,4 & 46,8 & 37,1 & 61 & 39,6 & 19,8 & 18,8 & 18,6 & 16,1 \\
\hline Zinc, mg/kg d.s. & 56,8 & 47,1 & 100,02 & 50,3 & 228,3 & 205,1 & 49,6 & 48,4 & 15,8 & 15,4 \\
\hline lron, mg/kg d.s. & 4256 & 3967 & 3744 & 3737 & 4065 & 3967 & 3352 & 3149 & 3068 & 2876 \\
\hline $\begin{array}{l}\text { Cooper, mg/kg } \\
\text { d.s. }\end{array}$ & 71,1 & 62,7 & 47,5 & 42,4 & 41,6 & 38,4 & 36,5 & 20,5 & 18,6 & 16,1 \\
\hline $\begin{array}{l}\text { Cadmium, mg/kg } \\
\text { d.s. }\end{array}$ & 1,16 & 0,71 & 0,68 & 0,19 & 0,25 & 0,18 & 0,19 & 0,22 & 0,15 & 0,12 \\
\hline
\end{tabular}

Table 4 Characterization of soil samples near the wastewater treatment plant of Slobozia- September 2015 campaign

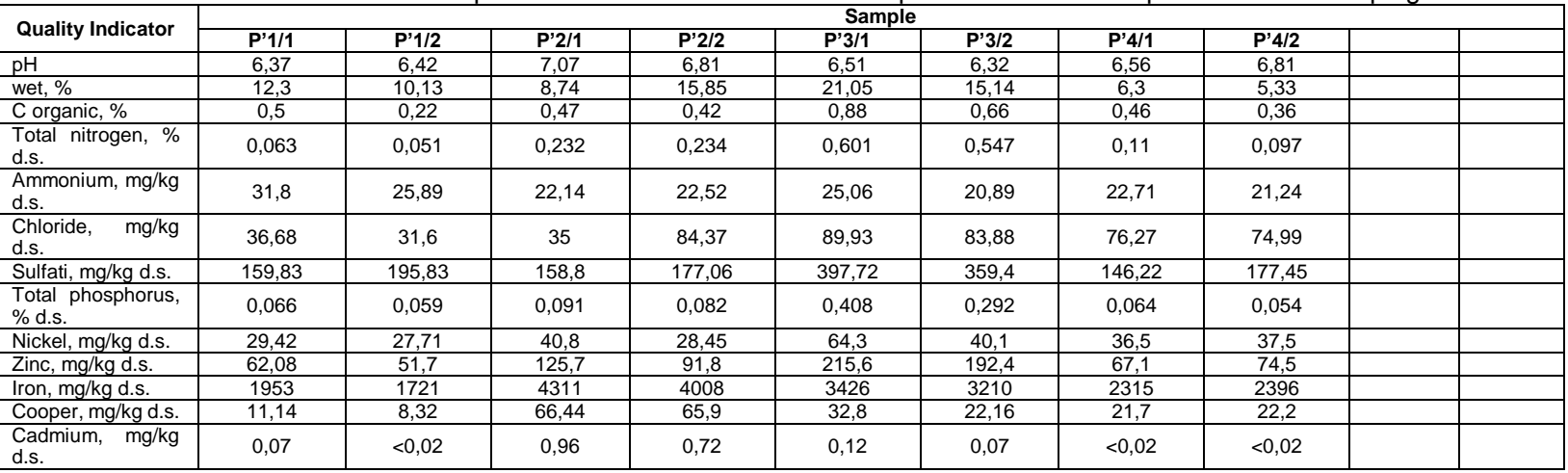

Table 5. Characterization of water samples near the wastewater treatment plant of Focsani

\begin{tabular}{|c|c|c|c|c|c|c|}
\hline \multirow[t]{2}{*}{ Quality Indicator } & \multicolumn{3}{|c|}{ July campaign 2015} & \multicolumn{3}{|c|}{ September campaign 2015} \\
\hline & $\begin{array}{c}\text { Effluent } \\
\text { discharge } \\
\text { station }\end{array}$ & $\begin{array}{l}\text { Putna river - } \\
\text { upstream } \\
\text { effluent } \\
\text { discharge } \\
\text { station }\end{array}$ & $\begin{array}{l}\text { Putna river } \\
- \\
\text { downstream } \\
\text { effluent } \\
\text { discharge } \\
\text { station } \\
\end{array}$ & $\begin{array}{l}\text { Effluent } \\
\text { discharge } \\
\text { station }\end{array}$ & $\begin{array}{l}\text { Putna river } \\
- \\
\text { upstream } \\
\text { effluent } \\
\text { discharge } \\
\text { station }\end{array}$ & $\begin{array}{l}\text { Putna river } \\
- \\
\text { downstream } \\
\text { effluent } \\
\text { discharge } \\
\text { station } \\
\end{array}$ \\
\hline $\mathrm{pH}$ & 7,44 & 6,83 & 7,58 & 7,65 & 7,78 & 7,71 \\
\hline $\mathrm{COD}, \mathrm{mg} \mathrm{O}_{2} / \mathrm{l}$ & 81 & 38,4 & 40,1 & 96 & 39 & 48 \\
\hline $\mathrm{BOD}, \mathrm{mg} \mathrm{O} / \mathrm{ll}$ & 25 & 12,7 & 11,1 & 25 & 11 & 14 \\
\hline Ammonium, mg/l & 1,68 & 0,58 & 0,59 & 0,96 & 0,78 & 0,75 \\
\hline Total nitrogen, $\mathrm{mg} / \mathrm{l}$ & $\frac{1,06}{6,21}$ & $\frac{0,06}{3,78}$ & 4,01 & $\frac{9,12}{9,12}$ & 4,02 & 5,8 \\
\hline Total phosphorus, $\mathrm{mg} / \mathrm{l}$ & 0,18 & $<0,02$ & 0,08 & 0,51 & 0,06 & 0,14 \\
\hline Nitrates, mg/l & 17,08 & 11,56 & 13,1 & 23,9 & 9,33 & 13,3 \\
\hline Nitrites, $\mathrm{mg} / \mathrm{l}$ & 0,8 & 0,5 & 0,6 & 0,69 & 0,21 & 0,29 \\
\hline Chloride, mg/l & 273,35 & 184,6 & 207,67 & 244,9 & 262,7 & 255,6 \\
\hline Sulfides, mg/l & $<0,04$ & $<0,04$ & $<0,04$ & $<0,04$ & $<0,04$ & $<0,04$ \\
\hline Residuu, mg/l & 966 & 630 & 728 & 934 & 856 & 822 \\
\hline Zinc, $\mathrm{mg} / \mathrm{l}$ & 0,16 & 0,08 & 0,09 & 0,023 & 0,009 & 0,018 \\
\hline Nickel, mg/l & 0,007 & 0,007 & 0,006 & 0,046 & 0,044 & 0,047 \\
\hline Cooper, mg/l & $<0,003$ & $<0,003$ & $<0,003$ & 0,006 & 0,006 & 0,005 \\
\hline Iron, $\mathrm{mg} / \mathrm{I}$ & 0,26 & 0,20 & 0,25 & 0,16 & 0,08 & 0,09 \\
\hline Cadmium, mg/l & 0,002 & 0,002 & 0,002 & $<0,001$ & $<0,001$ & $<0,001$ \\
\hline
\end{tabular}

Table 6. Characterization of water samples near the wastewater treatment plant of Slobozia

\begin{tabular}{|c|c|c|c|c|c|c|}
\hline \multirow[t]{2}{*}{ Quality Indicator } & \multicolumn{3}{|c|}{ July campaign 2015} & \multicolumn{3}{|c|}{ September campaign 2015} \\
\hline & $\begin{array}{l}\text { Effluent } \\
\text { discharge } \\
\text { station }\end{array}$ & $\begin{array}{l}\text { lalomita river } \\
\text { - upstream } \\
\text { effluent } \\
\text { discharge } \\
\text { station }\end{array}$ & $\begin{array}{c}\text { lalomita } \\
\text { river - } \\
\text { downstream } \\
\text { effluent } \\
\text { discharge } \\
\text { station }\end{array}$ & $\begin{array}{l}\text { Effluent } \\
\text { discharge } \\
\text { station }\end{array}$ & $\begin{array}{l}\text { lalomita } \\
\text { river - } \\
\text { upstream } \\
\text { effluent } \\
\text { discharge } \\
\text { station }\end{array}$ & $\begin{array}{l}\text { lalomita } \\
\text { river - } \\
\text { downstream } \\
\text { effluent } \\
\text { discharge } \\
\text { station }\end{array}$ \\
\hline $\mathrm{pH}$ & 7,86 & 7,82 & 7,84 & 7,27 & 6,63 & 6,77 \\
\hline $\mathrm{COD}, \mathrm{mg} \mathrm{O} \mathrm{O}_{2} / \mathrm{l}$ & 67,2 & 40 & 48,8 & $<30$ & $<30$ & $<30$ \\
\hline $\mathrm{BOD}, \mathrm{mg} \mathrm{\textrm {O } _ { 2 } / \mathrm { l }}$ & 21,8 & 12 & 18,1 & - & - & - \\
\hline Ammonium, mg/l & 1,19 & 0,97 & 1,03 & 0,696 & 0,71 & 0,696 \\
\hline Total nitrogen, mg/l & 4,87 & 1,91 & 1,66 & 4,37 & 4,4 & 4,86 \\
\hline Total phosphorus, mg/l & 0,97 & 0,097 & 0,13 & 0,98 & 0,187 & 0,173 \\
\hline Nitrates, $\mathrm{mg} / \mathrm{l}$ & 17,08 & 2,9 & 2,67 & 18,9 & 6,92 & 12,26 \\
\hline Nitrites, mg/l & 0,11 & 0,05 & 0,11 & 0,13 & 0,17 & 0,16 \\
\hline Chloride, mg/l & 266,2 & 237,8 & 222,7 & 253,8 & 216,5 & 213 \\
\hline Sulfides, mg/l & $<0,04$ & $<0,04$ & $<0,04$ & $<0,04$ & $<0,04$ & $<0,04$ \\
\hline Residuu, mg/l & 1252 & 732 & 730 & 1090 & 1028 & 1132 \\
\hline Zinc, mg/l & 0,14 & 0,2 & 0,16 & 0,11 & 0,08 & 0,09 \\
\hline Nickel, mg/l & 0,004 & 0,004 & 0,003 & 0,012 & 0,005 & 0,004 \\
\hline Cooper, mg/l & 0,004 & 0,003 & 0,003 & $<0,003$ & $<0,003$ & $<0,003$ \\
\hline Iron, mg/l & 0,37 & 0,27 & 0,28 & 0,29 & 0,21 & 0,18 \\
\hline Cadmium, mg/l & 0,003 & 0,004 & 0,003 & 0,009 & $<0,001$ & $<0,001$ \\
\hline
\end{tabular}

Highlighting compliance with the laws in force in the quality of environmental factors investigated in the vicinity of wastewater treatment sites was pursued by comparing the analytical determinations of samples with values stipulated in the provisions specific to each environmental components. 


\section{SOIL}

Comparing the quality parameter values determined in soil samples was done both with the values stipulated in Ord.756 / 1997 (for the rules indicators) [5] and blank values (for all indicators). Were highlighted the following:

\section{$>\quad$ Wastewater treatment plant of Focsani}

- $\quad \mathrm{pH}$ of soil samples indicates a neutral reaction soil near the station site - $\quad$ the indicators: $C$ organic, total nitrogen, total phosphorus had values that were above the values determined in the blank, but it not indicate pollution with these compounds

- considered heavy metals (nickel, zinc and copper), even if exceedings the proper normal values, were below the alert threshold values

The diagrams representation of the state of pollution in the area neighboring the WWTP Focsani, are presented for indicators: total phosphorus, organic carbon, total nitrogen.

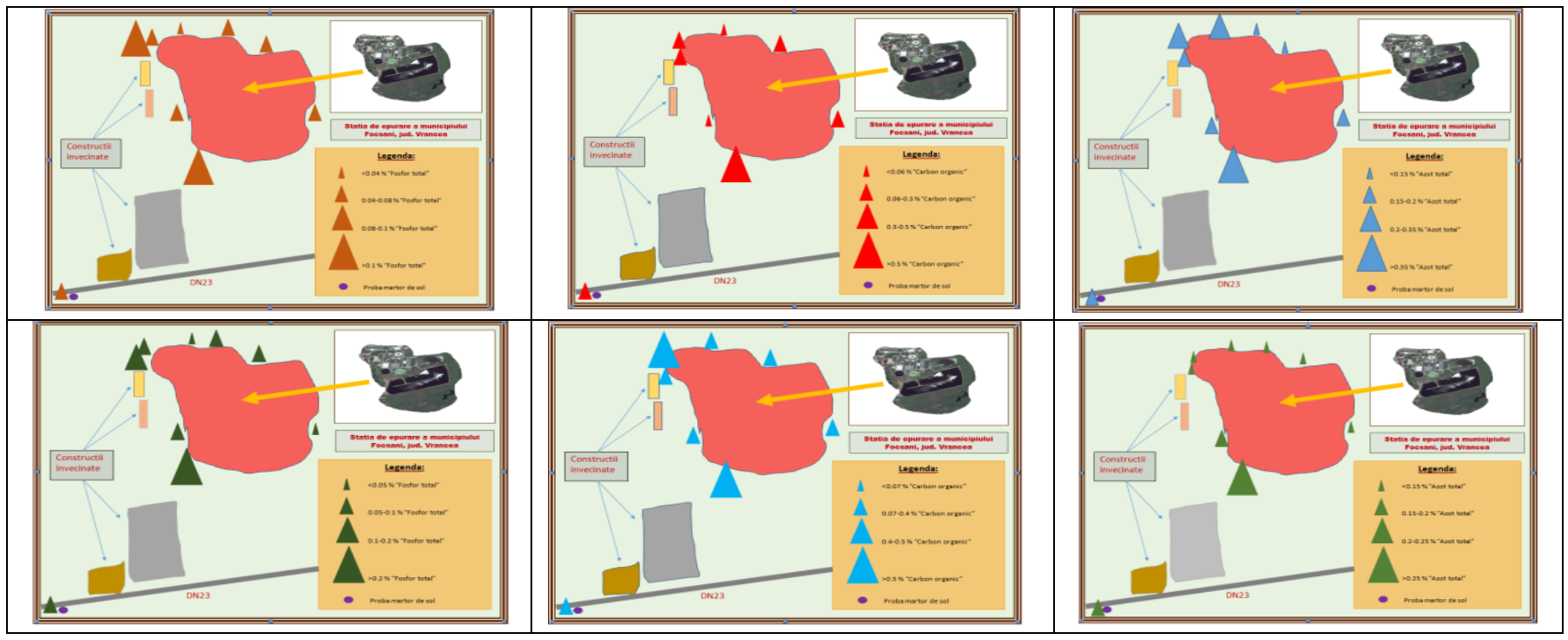

$>\quad$ Wastewater treatment plant of Slobozia

- $\quad \mathrm{pH}$ values for samples recorded in both campaigns, indicated a weak acidneutral reaction soil near the station

- $\quad$ the indicators: C organic, total nitrogen, total phosphorus had values that were above the values determined in the blank, but it they were located in areas of nonpolluted soils characteristic variation

- $\quad$ considered heavy metals (nickel, zinc and copper), even if exceedings the proper normal values, were below the alert threshold values

The diagrams representation of the state of pollution in the area neighboring the WWTP Focsani, are presented for indicators: total phosphorus, organic carbon, total nitrogen. 


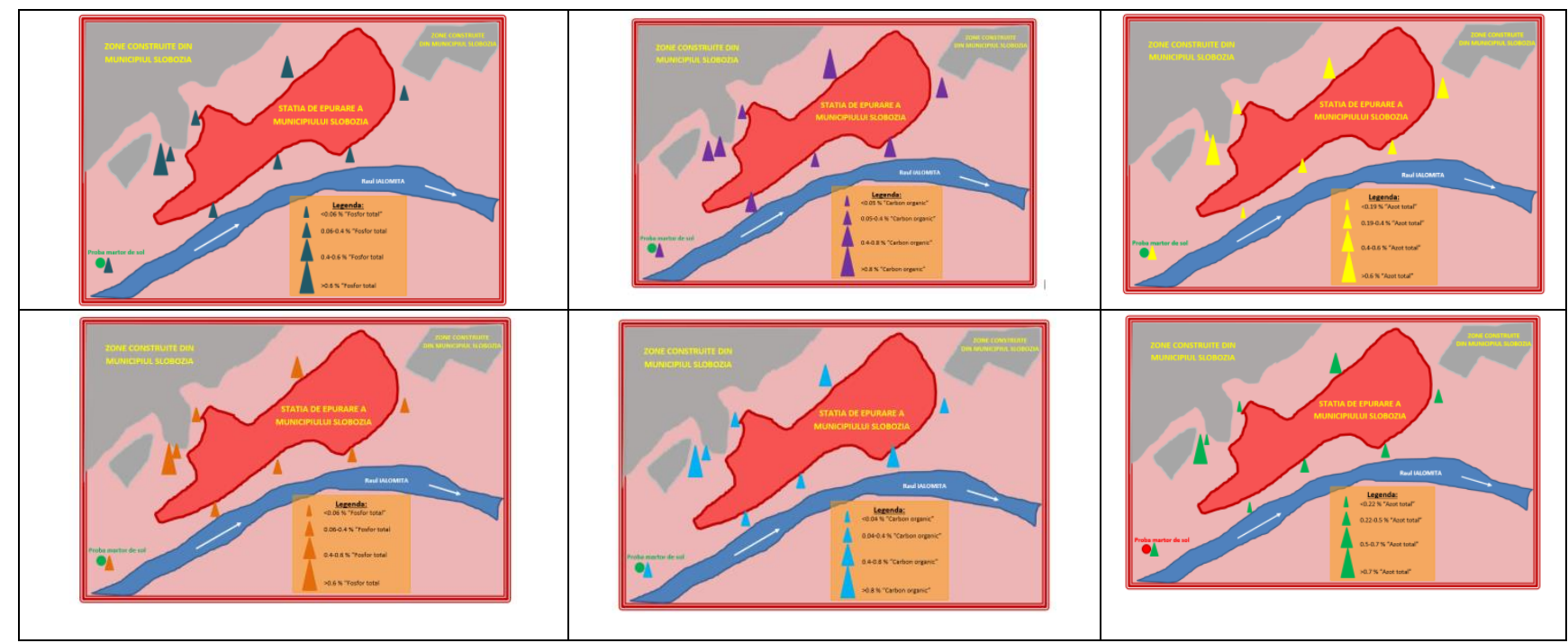

\section{WATER}

Values indicators analyzed in samples of treated effluent in both stations were located below the limits imposed by both environmental permits and by NTPA 001/2005 [6]. Therefore are discharged into emissaries natural receptors, from both WWTP,effluents with complying quality.

Analysis of the characteristics determined in samples of surface water in both campaigns investigation for both wastewater treatment plants have shown that, discharge of effluents station in natural emissaries (Putna river or lalomita river) have not led to changes in the state of quality to emissaries in upstream to downstream.

\section{Conclusions}

Investigation of environmental quality components in proximity of municipal sewage treatment plants Focsani and Slobozia has allowed to quantify the impact induced by the activities carried out in the two stations near the sites.

The results of characterize components of environmental soil and water, interpreted in accordance with environmental legislation specific and illustrating the state of pollution in the areas surrounding sewage treatment plants by presenting diagrams led to the conclusion that activities carried out in the sites did not induce negative effects in proximity.

\section{Acknowledgements}

Authors recognize the financial support from the National Nucleus Programme-2015

\section{References}

[1] V. Rojanschi, F. Bran, G. Diaconu, 2002, Protectia si ingineria mediului

[2] www.rowater.ro -2012, Brosura ape uzate pentru public

[3] www.greenly.ro - 2012, Revista de mediu

[4] B. Stanescu, G. Batrinescu, , A. Cuciureanu, L. Kim, D. Scradeanu, M. Scradeanu, 2014, Practical aspects of hydrogeological investigations and assessement of the environmental components pollution in urban areas. Case study, JEPE 15, no.3, 870-877

[5] Ord.756 al MAPPM, Reglementari privind evaluarea poluarii mediului, 1997

[6] HG 352/2005 - aprobarea normelor privind conditiile de descarcare in mediul acvatic a apelor uzate NTPA 001 - Valori-limita de incarcare cu poluanti a apelor uzate industriale si orasenesti in receptori naturali 